\title{
A fast non-local transform-domain method for seismic random noise attenuation
}

\author{
Sajjad AMANI ${ }^{1,2}$, Ali GHOLAMI ${ }^{1}$ and Hamidreza SIAH KOUHI ${ }^{1}$ \\ ${ }^{1}$ Institute of Geophysics, University of Tehran \\ ${ }^{2}$ Now, Dept. of Civil and Earth Res. Eng., Kyoto University
}

\begin{abstract}
All of the seismic data include different amounts of seismic random noises, even after doing a comprehensive seismic data processing. This results in lower signal to noise ratio (SNR) or in other word, lower quality of seismic data. Because of the time-consuming processes of methods for doing seismic random noise attenuation, data processing companies don't perform additional processing for attenuating of random noises after doing conventional methods like stacking and applying some filters. BUT, what about a very fast method which increases SNR both in pre-stacked and post-stacked data, significantly? Here, in this study we introduce an algorithm which is called 'Fast 3D Block Matching (F3DBM)' which combines the advantages of non-local and transform-domain denoising methods. This method has superior capability for preserving discontinuities presented in seismic data both qualitatively and quantitatively. We compare the ability of F3DBM with that of the state-of-the-art fast curvelet-based seismic denoising method for random noise attenuation both in pre-stacked and post-stacked data.
\end{abstract}

Key words: random noise, block matching, curvelet-based denoising method, pre-stacked and post-stacked seismic data denoising.

\section{INTRODUCTION}

The amount of signal to noise ratio, shows the quality of a seismic data set and is very important in accuracy of seismic interpretation for exploration of subsurface reservoirs. This amount is directly related to the level of noises through the data. Depending on the seismic acquisition process, these data are contaminated with different amount of random (incoherent) noises. The source of these noises can be anything and they are unpredictable and inevitably exist. Based on different theories, many methods have been proposed for attenuating of random noises ${ }^{1,2,3)}$ which can be collected in three main categorizes: $t-x$ domain methods (e.g. mean filters, median filters), transformed domain methods (e.g. f-x methods, f-k methods, wavelet methods), and combination methods (the combination of $1^{\text {st }}$ and $2^{\text {nd }}$ categorizes). In this study we propose a new and fast combination method based on 3DBM image processing algorithm ${ }^{3,4)}$ for attenuating random noises in pre-stacked and post-stacked seismic data. This method is called "Fast 3D Block Matching" and is a non-local transform-domain method. It combines the advantages of non-local and transform-domain denoising methods. It considers a seismic profile like an image and divides it into many blocks with the same size. Then, using the similarities between the blocks (intra- and inter-fragment correlation), attenuates the amount of random noises, significantly.

\section{METHOD}

Generally, the 3D block matching (3DBM) method consists of two main steps that are nearly equal except in some details. In both steps, 3DBM considers a noisy seismic cross section as an image. In the first step a basic estimation of the seismic profile is obtained by removing a part of random noise energy using hard thresholding. In the second step, using the basic estimation gained in the previous step and a Wiener filter, a significant part of random noises will be removed while preserving the features of the signal. Each of these two steps consist five successive subsections that are performed exclusively for each block. In the next parts we will introduce all of these subsections.

\section{(1) 3D grouping of similar blocks}

Let us consider the following model for seismic data $\mathrm{z}: \mathrm{X} \rightarrow \mathrm{R}$ 


$$
\mathrm{z}(\mathrm{x})=\mathrm{y}(\mathrm{x})+\mathrm{e}(\mathrm{x}), \quad \mathrm{x} \in \mathrm{X}
$$

where $\mathrm{z}$ is the noisy measured seismic data, $\mathrm{y}$ is the noise free seismic data of interest, e is zero-mean, white noise with variance $\sigma^{2}$ and $\mathrm{x}$ is the $2 \mathrm{D}$ spatial coordinate of the top left of the block that belongs to the domain $\mathrm{X} \subset Z$. A seismic section can be divided into many blocks $Z_{x}$ with the same size $\mathrm{N}$ $\times N$ (i.e. with the same number of samples). For every block we can find some blocks that are similar to it. When a block at $X_{R}$ is going to be processed, it is called "Reference Block". We use "matching" method for finding the blocks similar to a given reference one ${ }^{4,5}$. This method uses a pairwise testing the dissimilarity between the reference one and the candidate blocks located at different spatial locations. However, for speed up the block matching, a definite neighborhood will be chosen for these candidate blocks. The blocks whose dissimilarity from the reference one is smaller than a given threshold " $\tau$ " are considered similar and are subsequently grouped in a 3D array " $Z_{x_{R}}$ " (Figure 1), where $S_{x_{R}}$

$$
S_{x_{R}}=\left\{x \in X: d\left(Z_{x_{R}}, Z_{x}\right)<\tau\right\}
$$

is the positions of similar blocks to the reference block at position $x_{R}$ and $d$ means dissimilarity. It is important to say that the ordering of blocks in the group does not have a significant effect on the estimation ability of the algorithm but first in a group is the reference one ${ }^{4}$.

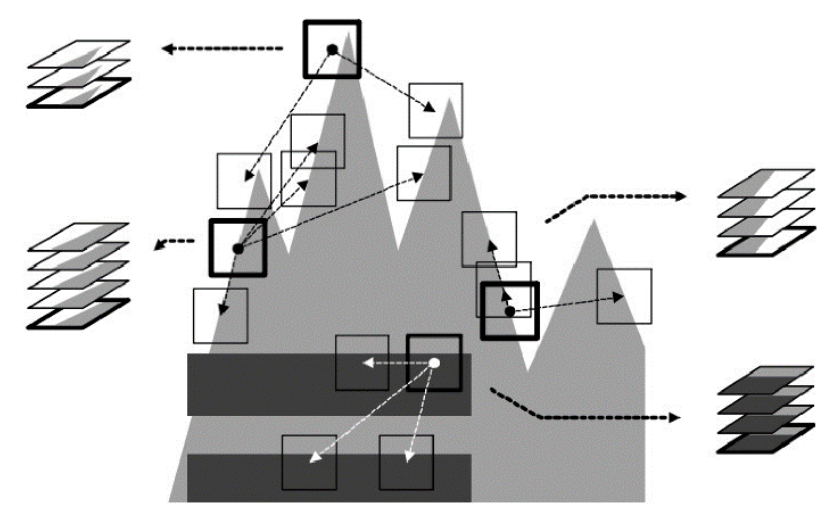

Figure 1: Grouping of the similar blocks in an image. The reference blocks are shown with thick borders ${ }^{4)}$.

\section{(2) 3D transformation of a group}

After providing a group, a 3D linear transformation is applied on it. The 3D transformation can take advantage of two kinds of correlations. First, the correlation between all of the samples of a block which is called intra-fragment correlation is calculated. Second, the correlation between the corresponding samples in all of the blocks in that group which is called inter-fragment correlation is calculated. The result is the production of a sparse representation of the noise free seismic data in the group. So, using an effective shrinkage operator, a significant part of the noise will be attenuated while preserving the features of the signal. One of the novelties in this method is applying a 2D transform, across the matched blocks and a 1D transform along the third dimension of a group (along which the blocks are stacked) instead of a 3D transform to the whole group. This results in the reduction of the complexity of applying a 3D transform. However, according to the reference ${ }^{4)}$, the sparsity improvement depends mainly on 1D transform for using the inter-fragment correlation among grouped blocks and the choice of 2D transform does not have a significant impact on the denoising performance. The $1 \mathrm{D}$ transform that is used in all of the procedures is a $1 \mathrm{D}-$ Haar transform.

\section{(3) Shrinkage of the transform spectrum}

In the previous subsection the grouped blocks transformed to another domain which produced sparse representation of the true signal. This signal is highly retrieved by attenuating the random noises by applying appropriate shrinkage operators which are a hard-thresholding and a Wiener filter in the steps 1 and 2, respectively. The Wiener filtering in step 2 uses the power spectrum of the basic estimate to filter the formed $3 \mathrm{D}$ groups which result in the significantly improvement of the final estimation of input data over the hard-thresholding used in the first step.

\section{(4) Inverse 3D transformation}

After applying mentioned shrinkage operators, it is time to return the filtered data to their first domain by using inverse transformation of the transforms that have been used in the previous subsections. Note that as mentioned above, instead of an inverse 3D transform, we use an inverse 1D with an inverse 2D transform. For easier understanding, the subsections 2, 3 and 4 are called Collaborative filtering. The results of collaborative filtering in step 1 and 2 for a reference block at $x_{R}$ are $Y_{x_{R}}^{\text {basic }}$ and $Y_{x_{R}}^{\text {final }}$, respectively, which are

$$
\begin{aligned}
& Y_{x_{R}}^{\text {basic }}=T_{3 D}^{-1}\left(\gamma\left(T_{3 D}\left(Z_{S_{x_{R}}^{1}}\right)\right)\right) \\
& Y_{x_{R}}^{\text {final }}=T_{3 D}^{-1}\left(W_{S_{x_{R}}^{2}}^{2} \cdot T_{3 D}\left(Z_{S_{x_{R}}^{2}}\right)\right)
\end{aligned}
$$

Where $T_{3 D}$ is the normalized 3D linear transform mentioned before and $T_{3 D}^{-1}$ is its inverse form, $\gamma$ 
is a hard-threshold operator with a given threshold, $Z_{S_{x_{R}}^{1}}$ is the group of noisy blocks in step 1 which their coordinates are $\mathrm{x} \in S_{x_{R}}^{1}, Z_{S_{x_{R}}^{2}}$ is the group of noisy blocks in step 2 which their coordinates are $\mathrm{x}$ $\in S_{x_{R}}^{2}, W_{S_{x_{R}}^{2}}$ is the empirical Wiener shrinkage coefficients of estimated blocks at $\mathrm{x} \in S_{x_{R}}^{2}$, and “." shows the dot production.

\section{(5) Return estimated blocks to their original locations}

In this subsection, the obtained $2 \mathrm{D}$ estimates of all grouped blocks will return to their original locations. But as mentioned before, because of doing 3DBM for each block, we obtain many estimates for each block which need to be combined. Aggregation is a particular averaging procedure which is exploited to take advantage of this redundancy. We compute the true estimates of each block by weighted averaging of all the obtained block estimates that are overlapping. Note that the aggregation process in step 1 and 2 , results in basic and final estimation of the whole noisy data, respectively.

\section{FAST AND EFFICIENT 3DBM}

In other to have a fast and efficient 3DBM method, we have to apply some constraints on 3DBM algorithm. Of course, the faster one has a little decreased denoising performance at the cost of significantly lower computational time. The constraints are:

1. Reduce the number of processed block using a step of given pixels in horizontal and vertical directions.

2. Reduce the complexity of the grouping, e.g. by choosing limited number of blocks per group and defining a certain neighborhood.

3. Reduce the complexity of applying transforms using the class of separable transforms.

4. Applying the aggregation efficiently in each step.

\section{RESULTS}

For demonstrating the ability of F3DBM, we applied this method on synthetic pre-stacked and post-stacked seismic data and compared them with the state-of-the-art Curvelet-based seismic denoising method (CT) which is one of the most powerful and fast methods in this area.

In Figure 2, the comparison of these two methods has been shown for a pre-stacked seismic data of salt dome with high amount of random noises (signal to noise ratio, SNR, of noisy data is $1 \mathrm{~dB}$ ). The result of F3DBM has higher SNR and is more than 9 times faster than fast CT method in this case. In addition, you can see a significant higher amount of artificial effects in the result of CT method (Fig. 2-c).

Finally, we compared the ability of both methods around the faults in figure 3 for a post-stacked data with high amount of random noises $(\mathrm{SNR}=1 \mathrm{~dB})$. This figure shows the superior capability of F3DBM for preserving faults and discontinuities in presented seismic data. In addition to higher quality (both qualitatively and quantitatively), F3DBM is more than 1.4 times faster than fast CT method in this case. Also, some artificial effects appeared in the results of CT method (Fig. 3-c) after attenuating of random noises.

\section{CONCLUSIONS}

In this study, we proposed F3DBM algorithm for fast seismic random noise attenuation and compared it with the state-of-the-art Curvelet-based seismic denoising method (CT) which is one of the most powerful and fast methods in this area. F3DBM combines the advantages of non-local and transform-domain denoising methods and uses the similarities through the signal for retrieving the amplitude of signal in a specific point in the $t-x$ domain, and because of this, it is more able to preserve discontinuities in the data. We showed this assertion in both synthetic and real seismic data. According to the results, in respect to the CT method, F3DBM has: 1. considerably lower execution time, 2. higher quality outputs both qualitatively and quantitatively, and 3. more stability for keeping the real signal while attenuating the random noises in the seismic data. As a consequence, it can be considered as an alternative method for random noise attenuation both in pre-stacked and post-stacked seismic data.

\section{REFERENCES}

1) Buades, A., Coll, B., and Morel, J. M., 2006, A review of image denoising algorithms, with a new One, Multiscale Modeling and Simulation, 4, 490-530.

2) Milanfar, P., 2013, A tour of modern image filtering: New insights and methods, both practical and theoretical, IEEE Signal Processing Magazine, 30, 106-128.

3) Amani, S., Gholami, A., Siah Kouhi, H., and Javaheri Niestanak, A., 2014, Seismic Random Noise Attenuation via 3D Block Matching, 
Master Thesis, University of Tehran.

4) Dabov, K., Foi, A., Katkovnik, V., and Egiazarian, K., 2007, Image denoising by sparse 3D transform-domain collaborative filtering, IEEE Transactions on Image Processing, 16,

a)

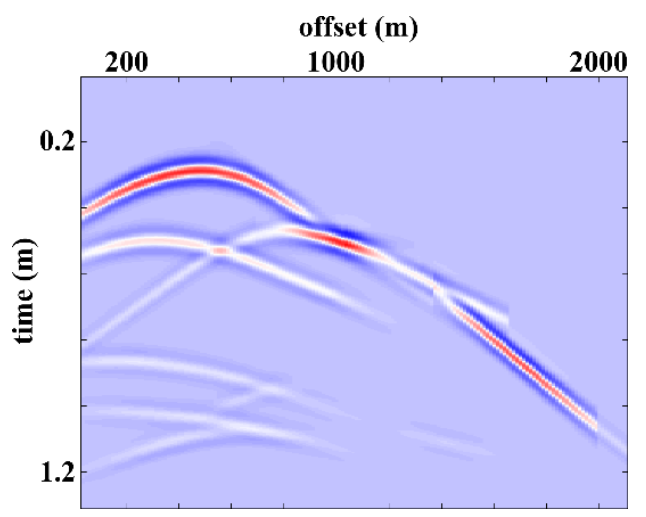

c)

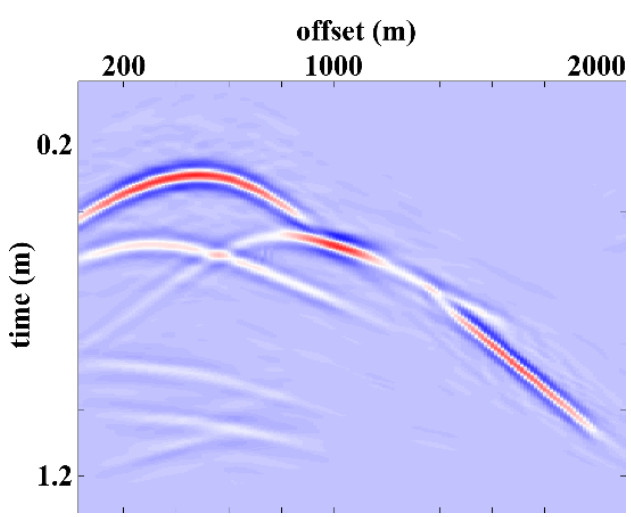

e)

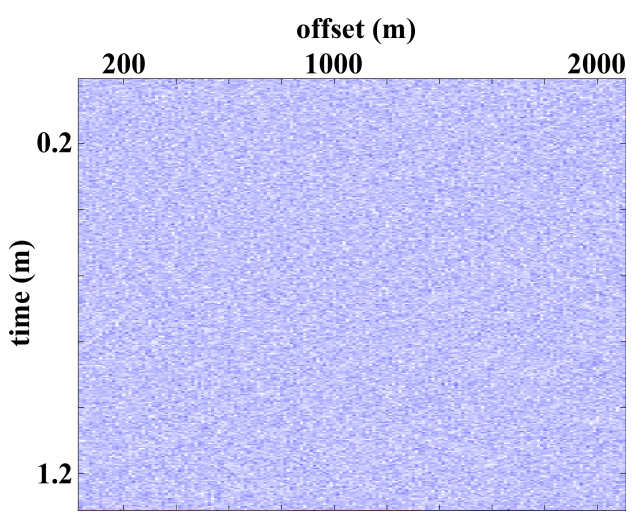

2080- 2095.

5) Jain, A., Murty, M., and Murty, P., 1999, Data clustering: A review, ACM Computing Surveys, 31, 264-323. b)

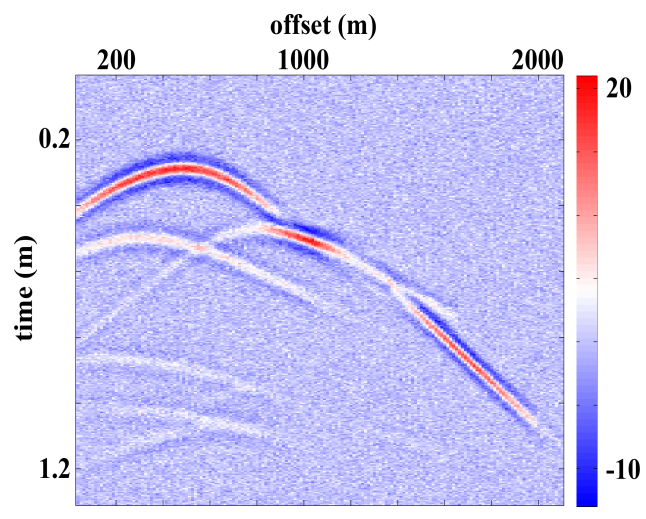

d)

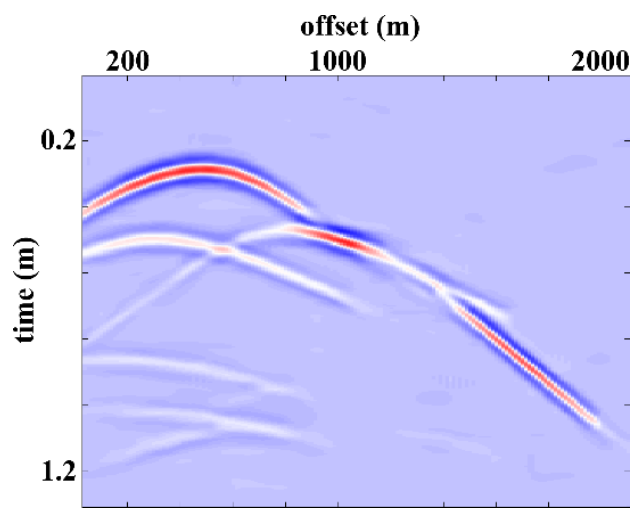

f)

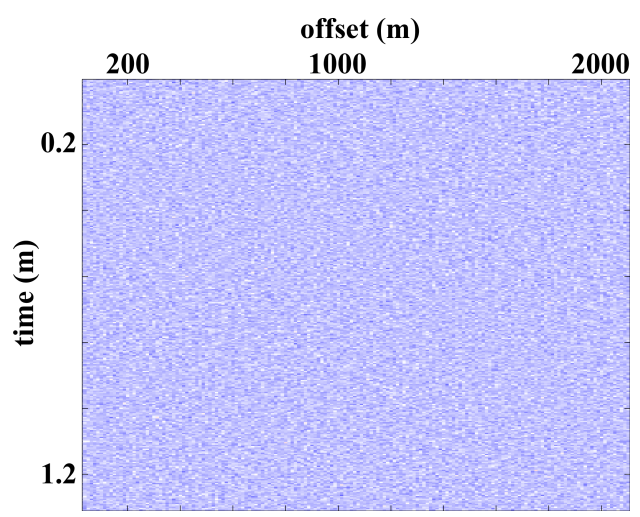

Figure 2: Comparison of the denoising effects and execution times for a pre-stacked seismic data with high amount of random noises. (a) Clean data $(400 \times 187)$, (b) noisy data with Gaussian white noise $(\mathrm{SNR}=1 \mathrm{~dB})$, (c) denoised result by fast $\mathrm{CT} \quad(\mathrm{SNR}=17.60 \mathrm{~dB}$, execution time $=1.190 \mathrm{sec}$ on $2 \mathrm{GHz}$ Core(TM)_i7-2630QM), (d) denoised result by F3DBM (SNR=18.61 dB, execution time= $0.128 \mathrm{sec})$, (e) removed noise section corresponding to (c), (f) removed noise section corresponding to (d). F3DBM is more than 9 times faster than fast CT method and has higher signal to noise ratio. High amount of artificial effects appeared in the results of CT method (c). 
a)

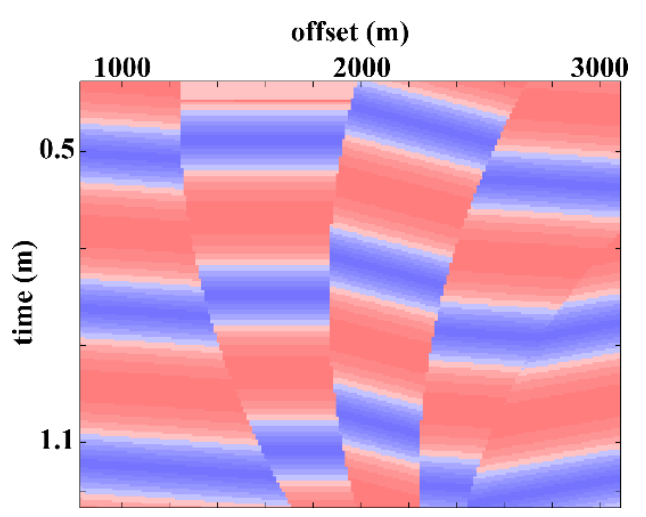

c)

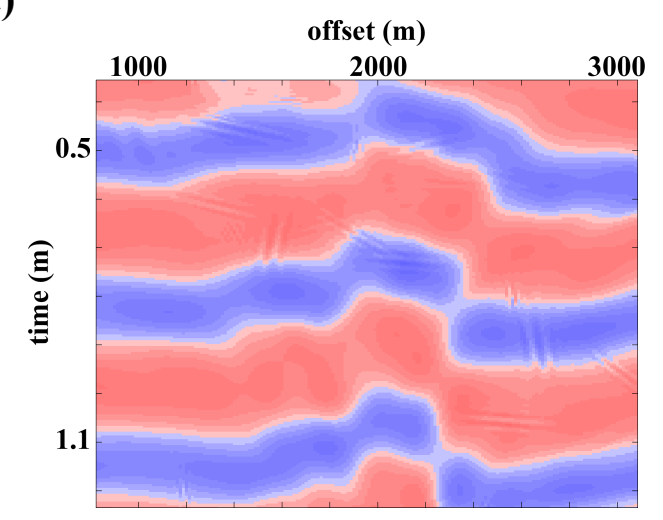

e)

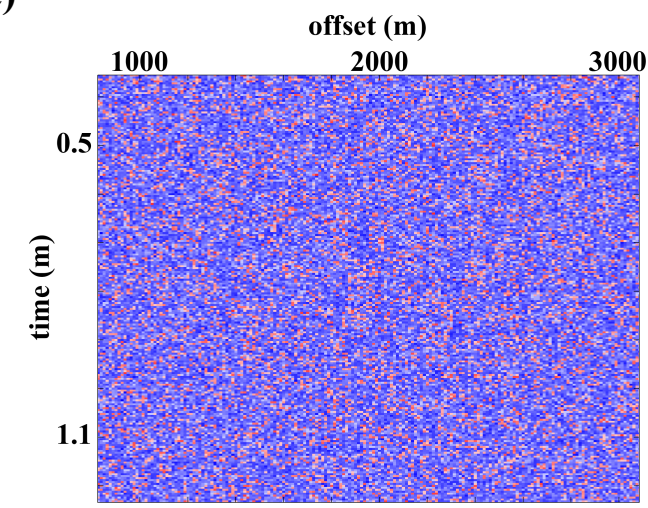

b)

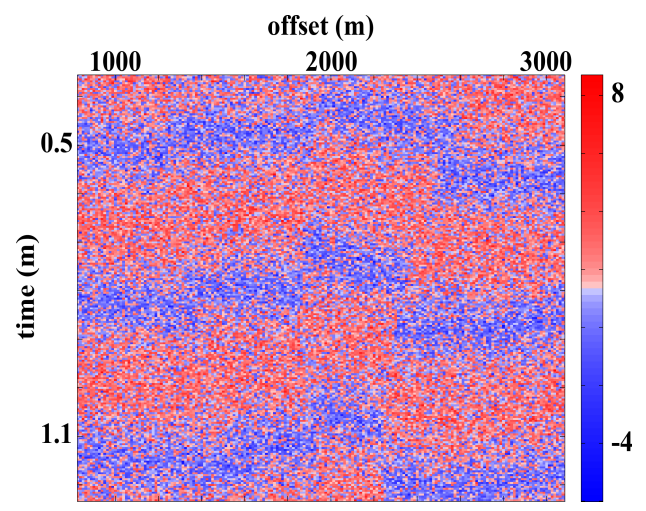

d)

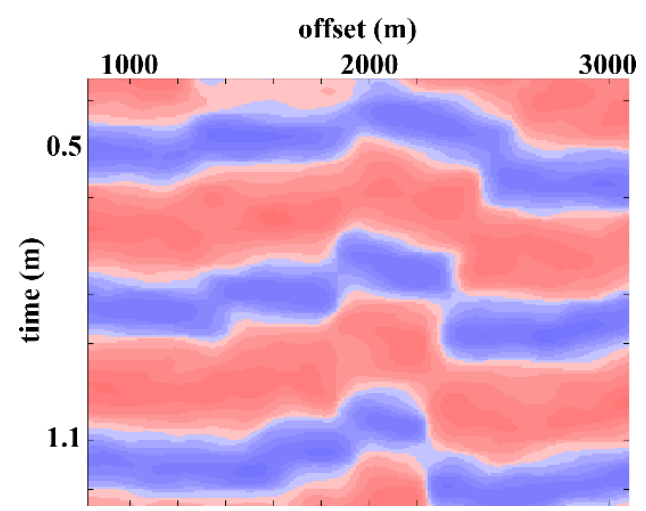

f)

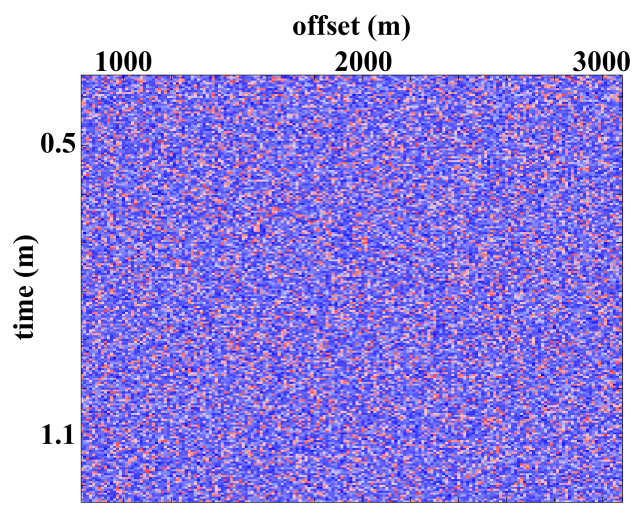

Figure 3: Comparison of the denoising effects and execution times around the faults for a post-stacked seismic data with highest amount of random noises. (a) Clean data $(512 \times 512)$, (b) noisy data with Gaussian white noise $(\mathrm{SNR}=1 \mathrm{~dB}),(\mathrm{c})$ denoised result by fast $\mathrm{CT}(\mathrm{SNR}=21.10 \mathrm{~dB}$, execution time $=3.20 \mathrm{sec}),(\mathrm{d})$ denoised result by F3DBM $(\mathrm{SNR}=21.43 \mathrm{~dB}$, execution time= $2.23 \mathrm{sec})$, (e) removed noise section corresponding to (c), (f) removed noise section corresponding to (d). F3DBM method is more than 1.4 times faster than fast CT method and has higher signal to noise ratio output. Also, F3dBM preserves signal more accurately around the faults which results in sharper boundaries around the faults (d). Some artificial effects appeared in the results of CT method (c). 